\title{
Displacement Talbot lithography: an alternative technique to fabricate nanostructured metamaterials
}

E. Le Boulbar, P. J. Chausse, S. Lis, P. Shields

E. D. Le Boulbar, P. J. P. Chausse, S. Lis, P. A. Shields, "Displacement Talbot lithography: an alternative technique to fabricate nanostructured metamaterials," Proc. SPIE 10248, Nanotechnology VIII, 102480Q (8 June 2017); doi: 10.1117/12.2265774

SPIE. Event: SPIE Microtechnologies, 2017, Barcelona, Spain 


\title{
Displacement Talbot lithography: an alternative technique to fabricate nanostructured metamaterials
}

\author{
E. D. Le Boulbar, P. J. P. Chausse, S. Lis and P. A. Shields* \\ Department of Electrical and Electronic Engineering, Claverton Down, University of Bath, Bath, \\ BA2 7AY, UK
}

\begin{abstract}
Nanostructured materials are essential for many recent electronic, magnetic and optical devices. Lithography is the most common step used to fabricate organized and well calibrated nanostructures. However, feature sizes less than $200 \mathrm{~nm}$ usually require access to deep ultraviolet photolithography, e-beam lithography or soft lithography (nanoimpriting), which are either expensive, have low-throughput or are sensitive to defects. Low-cost, high-throughput and low-defectdensity techniques are therefore of interest for the fabrication of nanostructures. In this study, we investigate the potential of displacement Talbot lithography for the fabrication of specific structures of interest within plasmonic and metamaterial research fields. We demonstrate that nanodash arrays and 'fishnet'-like structures can be fabricated by using a double exposure of two different linear grating phase masks. Feature sizes can be tuned by varying the exposure doses. Such lithography has been used to fabricate metallic 'fishnet'-like structures using a lift-off technique. This proof of principle paves the way to a low-cost, high-throughput, defect-free and large-scale technique for the fabrication of structures that could be useful for metamaterial and plasmonic metasurfaces. With the development of deep ultraviolet displacement Talbot lithography, the feature dimensions could be pushed lower and used for the fabrication of optical metamaterials in the visible range.
\end{abstract}

Keywords: Displacement Talbot Lithography, nanolithography, lift-off, metamaterials, metasurface, plasmonic, Talbot effect

\section{INTRODUCTION}

Artificially nanostructured materials with novel electromagnetic and optical properties, currently referred as metamaterials, are being extensively researched due to their extraordinary properties. Arrays of holes with a small periodicity, dashes and "fishnet" structures have been widely investigated and are of great interest within plasmonic and metamaterial research fields. These type of structures have been used to fabricate plasmonic metasurfaces that enable waveplates ${ }^{1,2}$, linear-to-circular polarization converters ${ }^{3}$, helicity-preserving omnidirectional mirrors ${ }^{4}$, and for biosensing applications ${ }^{5-7}$. Multilayer metal-dielectric-metal metamaterials based on these structures have been used to generate second or third harmonic wavelengths using their non-linear properties ${ }^{8-11}$ and create negative-index metamaterials (NIMs) from arrays of dashes ${ }^{12}$ and fishnets ${ }^{13-15}$.

Most of the early studies have used e-beam lithography or a focused ion beam in order to fabricate, develop and investigate the properties of these new materials. The fabrication of metamaterials at NIR or visible optical wavelengths requires small periodicities of $600 \mathrm{~nm}$ down to $300 \mathrm{~nm}$ and feature sizes of 200 to sub-100 nm. This typical feature size for infrared and visible range metamaterials is smaller than the resolution of conventional photolithography and requires a nanofabrication process with 100 or sub-100 nm resolution. High precision, high throughput and low cost manufacturing of such optical NIMs is therefore challenging due to the low dimensions that are required. Since the early research stage and first demonstration of these NIMs structures by different groups in 2005-2006 ${ }^{13-15}$, many groups have successfully demonstrated large-area fabrication processes. Fabrication of large-area fishnet structures has been realized using a wide range of techniques ${ }^{16}$, like deep-UV lithography ${ }^{17,18}$, nanoimprint lithography ${ }^{19,20}$ and interference lithography ${ }^{2,21}$.

Recently, a new lithography technique, taking advantage of the Talbot effect, has shown that high-resolution sub-micron patterns can be achieved by moving the sample position through one Talbot period ${ }^{22,23}$. The conventional Talbot effect occurs when a periodic mask is illuminated by a plane-wave. The three-dimensional interference pattern

Nanotechnology VIII, edited by lon M. Tiginyanu, Proc. of SPIE Vol. 10248, 102480Q

(c) The Authors. Published under a Creative Commons Attribution CC-BY 3.0 License · doi: 10.1117/12.2211588 
created below the mask is called Talbot carpet. Although this effect has been known since 1836, its low depth-of-field has meant that it has not been used for practical applications in semiconductor technology, especially for submicron features. A new method, called Displacement Talbot Lithography (DTL) and commercialized by Eulitha, unlocks the use of the Talbot effect and offers non-contact wafer-scale fabrication of periodic patterns of sub-micron features.

Displacement Talbot lithography, using a $375 \mathrm{~nm}$ source, and hence conventional i-line resists, allows the fabrication of linear gratings with submicron structure and periodicities as low as $250 \mathrm{~nm}$, owing to the doubling of the mask period frequency for linear gratings ${ }^{22}$. Smaller feature sizes are possible with the recent development of systems with a deep UV source $(\lambda=266 \mathrm{~nm})^{23}$. For linear gratings, one of the main features of DTL is that the frequency of the mask will be doubled on the wafer, i.e. using a $800 \mathrm{~nm}$ period linear grating mask will produce a $400 \mathrm{~nm}$ linear grating on the wafer. A second feature is the ability to control the size of the feature on the wafer through varying the exposure dose.

In this article, the potential of displacement Talbot lithography to fabricate nanostructures such as metallic nanodashes and 'fishnet' like structures from different linear gratings will be explored. (400 nm, and $600 \mathrm{~nm}$ ) by using a double exposure process. Two different grating masks, perpendicular to each other, are used to expose sequentially a single photoresist-covered sample prior to development. The capability of the DTL process will be demonstrated and compared with a model in order to explain the tunability of the resist linewidth that the DTL offers, taking the $400 \mathrm{~nm}$ linear gratings as an example. The fabrication of dashes and fishnet structures in photoresist and the range of dimensions that one can obtain by varying the exposure dose will be demonstrated and discussed. Finally, the lift-off process used to obtain metallic fishnets of various dimensions will be shown.

\section{EXPERIMENTAL SECTION}

An HSQ spin-on-glass layer (Hydrogen silsesquioxane, DOW Corning XR-1541) was spun on a $50 \mathrm{~mm}$ silicon (100) wafer to obtain a layer thickness of $120 \mathrm{~nm}$. The layer was baked on a hot plate at $450^{\circ} \mathrm{C}$ for 10 minutes. A bottom antireflective layer (BARC) (Brewer science - Wide 8C) was spun on top of the HSQ layer to obtain a layer thickness of $90 \mathrm{~nm}$. The layer was baked on a hot plate for $1 \mathrm{~min}$ at $80^{\circ} \mathrm{C}$ and $1 \mathrm{~min}$ at $210^{\circ} \mathrm{C}$. A $250 \mathrm{~nm}$ layer of high contrast positive resist (Dow ${ }^{\circledR}$ Ultra-i 123 diluted with Dow ${ }^{\circledR} \mathrm{EC} 11$ solvent) was spun on top of the BARC and cured at $90^{\circ} \mathrm{C}$ for $90 \mathrm{sec}$. For the negative resist, a layer of $350 \mathrm{~nm}$ (AZ® 15 NXT diluted with AZ® Edge Bead Remover (EBR) solvent with a 7:12 ratio by weight) was spun on top of the BARC and cured at $110^{\circ} \mathrm{C}$ for $45 \mathrm{sec}$.

PhableR (Eulitha) Displacement Talbot lithography equipment was used to expose the resist ${ }^{22,24}$. The gap between the mask and the wafer was set to $200 \mu \mathrm{m}$. The range of displacement of the wafer was set to two Talbot lengths, i.e. $6.4 \mu \mathrm{m}$ and $15 \mu \mathrm{m}$ for the $800 \mathrm{~nm}$ and the $1.2 \mu \mathrm{m}$ linear grating mask, respectively. The filling factor on the phase mask is approximately $62 \%$. The Talbot length was calculated using equation (1).

$$
T_{L}=\frac{\lambda}{1-\sqrt{1-\frac{\lambda^{2}}{p^{2}}}}(1)
$$

A coherent $375 \mathrm{~nm}$ illumination source with an energy density of $1 \mathrm{~mW} . \mathrm{cm}^{-2}$ was used. The energy density was controlled in real-time which allow an accurate and reliable exposure dose. After exposure, both samples with negative and positive resist were post-baked for $90 \mathrm{sec}$ at $120^{\circ} \mathrm{C}$. Samples with positive resist were dipped in MF-CD-26 developer for $90 \mathrm{sec}$. Samples with negative resist were dipped in AZ 726 developer for $40 \mathrm{sec}$. They were then rinsed with deionized water and dried with nitrogen gas.

\subsection{Effect of the exposure dose on feature sizes}

We investigated the impact of the dose exposure on linewidth, starting from an exposure dose of $50 \mathrm{~mJ} . \mathrm{cm}^{-2}$ up to $80 \mathrm{~mJ}_{\mathrm{cm}}{ }^{2,}$, with $5 \mathrm{~mJ} . \mathrm{cm}^{-2}$ incremental steps. The planar secondary electron (SE) scanning electron microscope (SEM) images of the pattern after development show a decrease of the linewidth with increased exposure dose (Fig. 1). The resist linewidths were extracted from the SE images and were found to decrease linearly, from $240 \mathrm{~nm}$ to $120 \mathrm{~nm}$, as the exposure dose increases from 50 to $80 \mathrm{~mJ} . \mathrm{cm}^{-2}$ (Fig. 2). The resist linewidth is found to change at a rate of $-3.75 \mathrm{~nm}$ per $\mathrm{mJ} . \mathrm{cm}^{-2}$. The linewidth of the resist can be carefully tuned controlled by adjusting the exposure dose. 


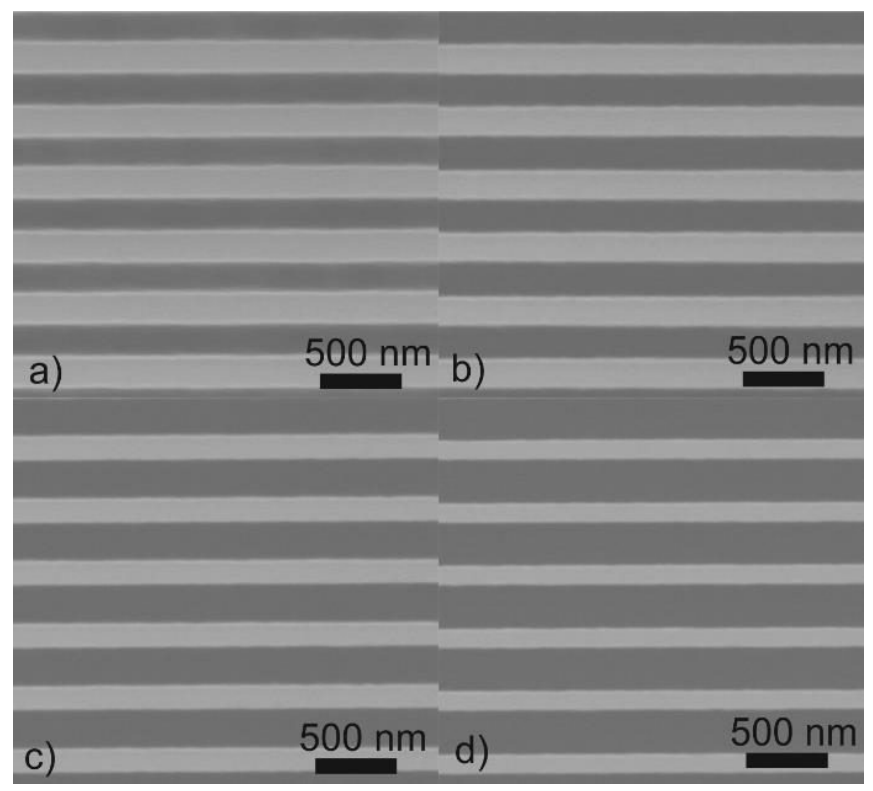

Figure 1: Secondary electron SEM images of $400 \mathrm{~nm}$ period linear gratings obtained in positive resist for an exposure dose of a) 50 $\mathrm{mJ} . \mathrm{cm}^{-2}$ b) $60 \mathrm{~mJ} . \mathrm{cm} \mathrm{c)} 70 \mathrm{~mJ} . \mathrm{cm}^{-2}$ and d) $80 \mathrm{~mJ} . \mathrm{cm}^{-2}$.

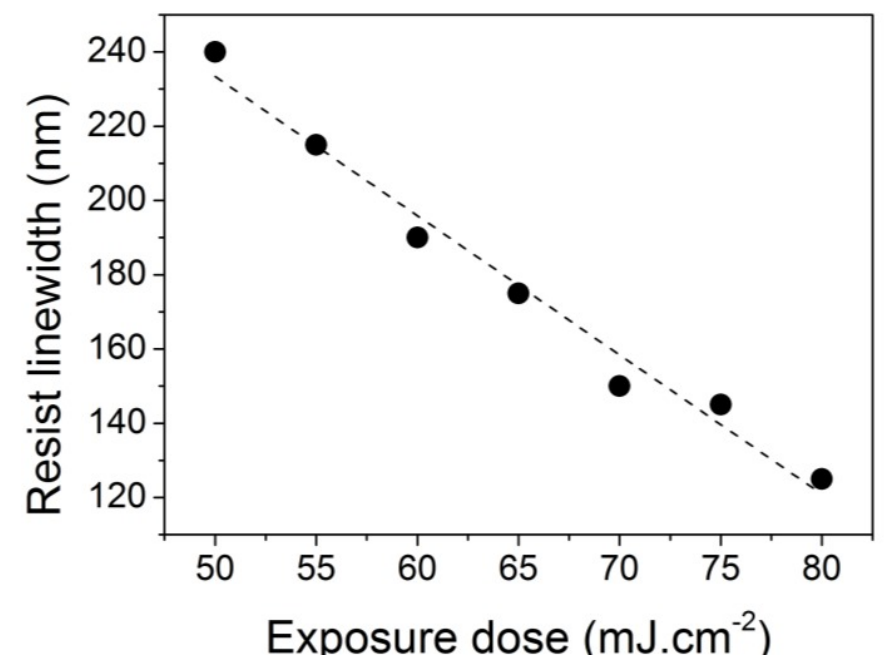

Figure 2: Resist linewidth obtained in a $800 \mathrm{~nm}$ thick positive resist (Ultra i123) as a function of the exposure dose for a $800 \mathrm{~nm}$ period linear grating mask. Linear slope is calculated as $-3.75 \mathrm{~nm} \cdot \mathrm{mJ}^{-1} \cdot \mathrm{cm}^{2}$

\subsection{Fabrication of dashes}

In order to create dash features, positive photoresist was first exposed using a $800 \mathrm{~nm}$ linear grating phase mask, with an exposure dose of $50 \mathrm{~mJ} . \mathrm{cm}^{-}{ }^{2}$. The sample was than rotated by $90^{\circ}$ and a second exposure was performed using a $1.2 \mu \mathrm{m}$ linear grating phase mask. The energy of the second exposure was varied from 20 to $30 \mathrm{~mJ} . \mathrm{cm}^{-2}$. After development, resist dashes of various dimensions were obtained on the wafer (Fig. 3). The periodicities in the two perpendicular directions, $a$ and $b$ were $400 \mathrm{~nm}$ and $600 \mathrm{~nm}$ respectively. As the alignment between the first and second exposure was made by eye, the dashes were not always aligned perfectly perpendicularly (e.g. in Fig. 3b). Suitable alignment marks could solve this issue. 


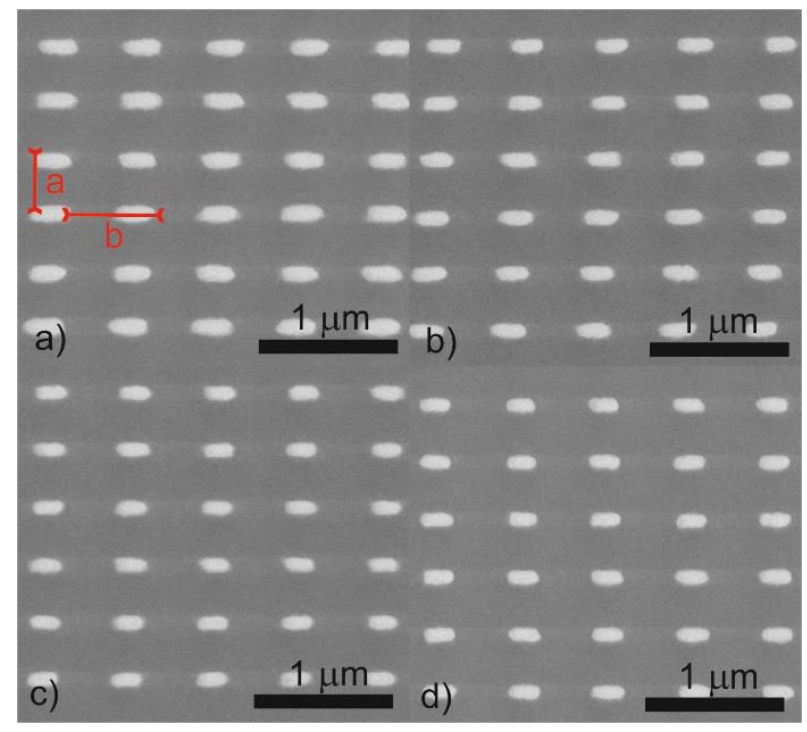

Figure 3: Dashes of positive resist obtained after double exposure for a dose of $50 \mathrm{~mJ} . \mathrm{cm}^{-2}$ for the $800 \mathrm{~nm}$ period linear grating pattern and a) $20 \mathrm{~mJ} . \mathrm{cm}^{-2}$ b) $22.5 \mathrm{~mJ} . \mathrm{cm}^{-2}$ c) $25 \mathrm{~mJ} . \mathrm{cm}^{-2}$ and d) $30 \mathrm{~mJ}_{\mathrm{cm}} \mathrm{cm}^{-2}$ for the $1.2 \mu \mathrm{m}$ period linear grating

The measured width (blue square) and length (red circle) of the dashes are plotted in Fig. 4. The width and length of the resist dashes are found to decrease with the increase of the second exposure dose (with $1.2 \mu \mathrm{m}$ period linear grating). The variation of dimension in width is much smaller ( $\Delta$ width $\sim 20 \mathrm{~nm}$ ) than the variation of length ( $\Delta$ length $\sim 60 \mathrm{~nm}$ ). From 20 to $25 \mathrm{~mJ} \mathrm{~cm}^{-2}$, a linear decrease is seen in both width and length, from $120 \mathrm{~nm}$ to $100 \mathrm{~nm}$ in width $\left(-4 \mathrm{~nm} / \mathrm{mJ}-1 . \mathrm{cm}^{2}\right)$ and from $270 \mathrm{~nm}$ to $220 \mathrm{~nm}$ in length $\left(-10 \mathrm{~nm} / \mathrm{mJ} \cdot \mathrm{cm}^{2}\right)$. The width of the dashes is found to be much smaller than the linewidth of $240 \mathrm{~nm}$ that was obtained for a single illumination of $50 \mathrm{~mJ} . \mathrm{cm}^{-2}$ (see Fig. 2), suggesting that the second illumination has significantly impacted the illumination of the resist exposed with the first linear grating mask. Between 25 and $30 \mathrm{~mJ} . \mathrm{cm}^{-2}$, there is only a small change in dash length and width. For $35 \mathrm{~mJ} . \mathrm{cm}^{-2}$, the resist was completely exposed so that no pattern was seen on the wafer.

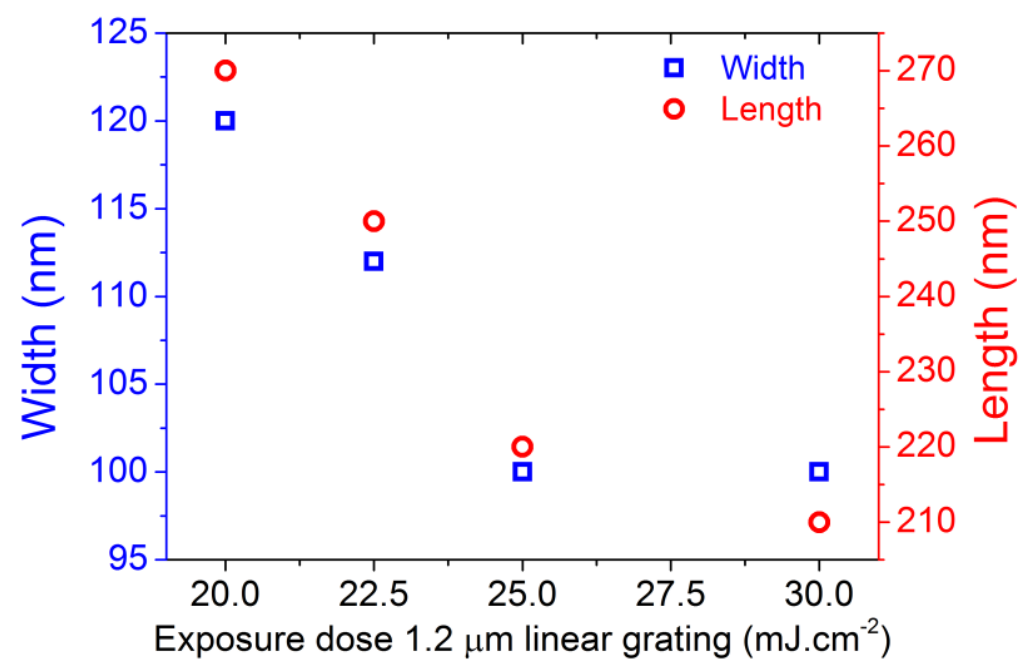

Figure 4: Length and width of the nanodashes obtained for a fixed exposure dose of $50 \mathrm{~mJ} . \mathrm{cm}^{-2}$ for the $800 \mathrm{~nm}$ period linear grating mask as a function of the exposure dose for the $1.2 \mu \mathrm{m}$ period linear grating mask. 


\subsection{Modelling of the aerial intensity distribution and resist development}

Modelling was performed in order to understand the results obtained, using two different linear grating phase masks and rotating the sample by $90^{\circ}$ between each exposure. A $800 \mathrm{~nm}$ period and a $1.2 \mu \mathrm{m}$ period grating phase mask that have a filling factor of $62 \%$ were used in the modelling to match the gratings used in our experiments. Fig. $5 \mathrm{a}$ and $5 \mathrm{~b}$ display a normalised aerial pattern, which represents the energy of the light below the mask integrated over one Talbot length at a distance of $200 \mu \mathrm{m}$ from the mask, for a $800 \mathrm{~nm}$ and a $1.2 \mu \mathrm{m}$ period linear grating mask. Fig. $5 \mathrm{c}$ and $5 \mathrm{~d}$ show corresponding cross-sections through the intensity distributions across the gratings and highlight that the intensity of the two alternating families of peaks are not equal. The $1^{\text {st }}$ family is slightly more intense than the $2^{\text {nd }}$ in the case of the 800 $\mathrm{nm}$ period linear grating while the opposite is found in the case of the $1.2 \mu \mathrm{m}$ period linear grating. In both cases, an intensity background equivalent to $\sim 0.2$ is present. This intensity background will have an impact on the illumination of the entire resist. This explains why an increase of energy on the $1.2 \mu \mathrm{m}$ period linear grating led to a decrease in the width of the dashes. It also explains why a dose of $50 \mathrm{~mJ} . \mathrm{cm}^{-2}$ for the first exposure produces dashes of width between 100 to $120 \mathrm{~nm}$ if followed by a second exposure, whilst a $240 \mathrm{~nm}$ grating linewidth is obtained for a single exposure.

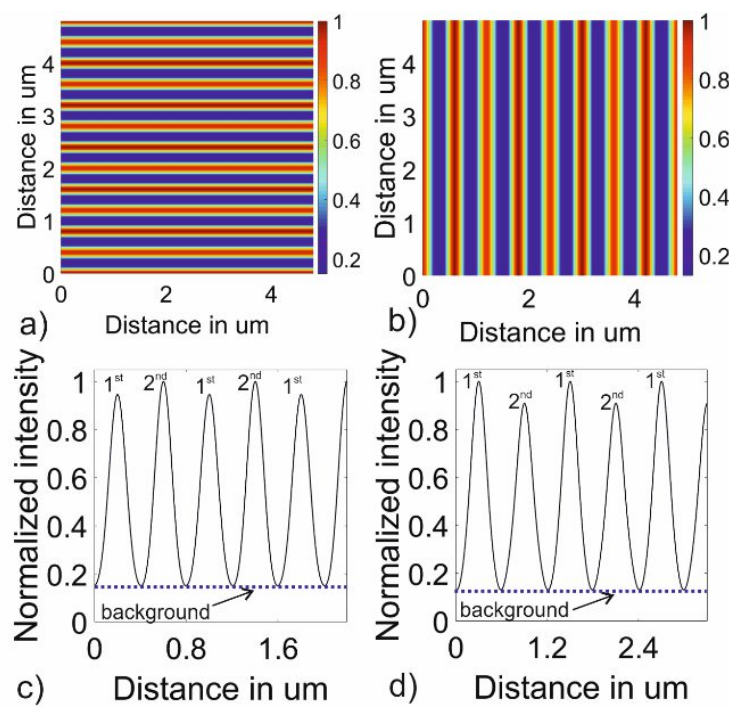

Figure 5: Aerial intensity image of the Talbot carpet integrated over one Talbot length for a) a $800 \mathrm{~nm}$ period linear grating phase mask b) a $1.2 \mu \mathrm{m}$ period linear grating mask. Normalized intensity of the energy obtained below the mask at a distance of $200 \mu \mathrm{m}$ for c) a $800 \mathrm{~nm}$ period d) $1.2 \mu \mathrm{m}$ linear grating mask.

Combining both exposures, a new aerial intensity distribution was obtained. The exposure dose of the $800 \mathrm{~nm}$ period linear grating was fixed at $170 \mathrm{~mJ} . \mathrm{cm}^{-2}$ whilst the exposure dose of the $1.2 \mu \mathrm{m}$ period linear grating was varied, with values of $30 \mathrm{~mJ} . \mathrm{cm}^{-2}$ and $70 \mathrm{~mJ} . \mathrm{cm}^{-2}$ (Fig.6a and 6b). The results from the development of the resist after exposure shows that length of the dashes will be reduced substantially by increasing the energy while the width change is relatively small (about $7 \mathrm{~nm}$ ). The asymmetry of the dashes, seen especially in Fig. $6 \mathrm{c}$ can be attributed to the variation of relative intensity between the primary and the secondary pattern. A more appropriate mask filling factor could be designed in order to obtain the same energy for both the primary and secondary pattern. This effect was not seen in our experiments. 

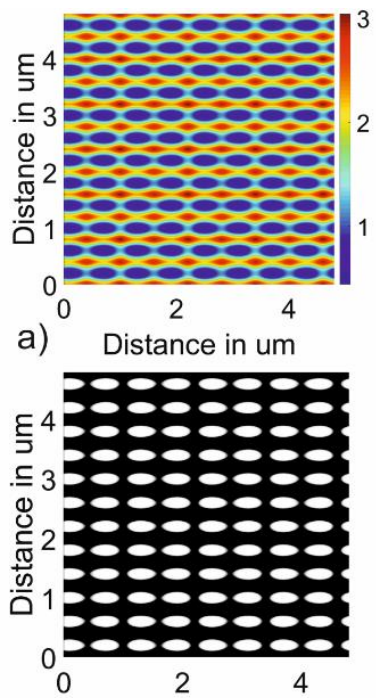

C)

Distance in um

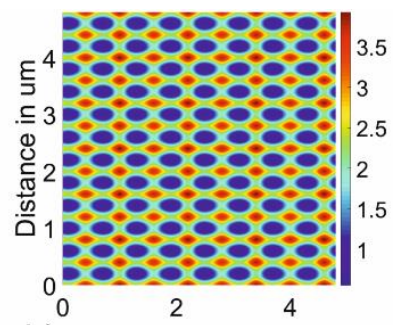

b) Distance in um

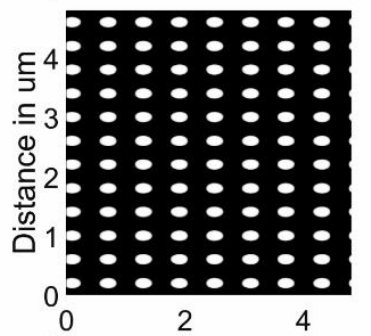

d) Distance in um

Figure 6: Aerial intensity distribution obtained for the combination of a $800 \mathrm{~nm}$ and $1.2 \mu \mathrm{m}$ linear grating mask rotated by $90^{\circ}$ with respective exposure doses of a) $170 / 30 \mathrm{~mJ} . \mathrm{cm}^{-2}$ and b) $170 / 70 \mathrm{~mJ} . \mathrm{cm}^{-2}$. Corresponding resist patterns expected after development for a double exposure for doses: c) $170 / 30 \mathrm{~mJ} . \mathrm{cm}^{-2}$ and d) $170 / 70 \mathrm{~mJ} . \mathrm{cm}^{-2}$.

\subsection{Creation of a lift-off profile}

The $250 \mathrm{~nm}$ thick resist was used as an etch mask and transferred into the underlying HSQ layer using a plasma dry etch step in order to create an undercut lift-off profile. $\mathrm{CHF}_{3}$ plasma was used with the following parameters: a pressure of $8 \mathrm{mTorr}$, an RF power of $50 \mathrm{~W}$, an ICP power of $300 \mathrm{~W}$ and a $\mathrm{CHF}_{3}$ flow of $25 \mathrm{sccm}$. The temperature was set to $20^{\circ} \mathrm{C}$ and $1 \mathrm{~min}$ etch, 1 min cooling steps were introduced in order to maintain the sample temperature. The etching rates for this recipe were measured to be $45 \mathrm{~nm} \cdot \mathrm{min}^{-1}$ for the resist and the BARC and $48 \mathrm{~nm} \cdot \mathrm{min}^{-1}$ for the HSQ. The samples were etched for $5 \mathrm{~min}$ to allow the etching of $90 \mathrm{~nm}$ of the BARC layer and $120 \mathrm{~nm}$ of HSQ. The planar SE image taken after the 5 min etching steps highlight the dimensions of the dashes were not fundamentally modified by the etching step (Fig. 7a). After this plasma etch step, the sample was dipped in a solution of Buffered Oxide Etch (BOE) 100:1 at $20^{\circ} \mathrm{C}$ for $35 \mathrm{sec}$. The SE cross-section image in Fig. $7 \mathrm{~b}$ displays the profile of the obtained structure after the $\mathrm{CHF}_{3}$ and the BOE steps and shows a clear undercut can has been obtained.

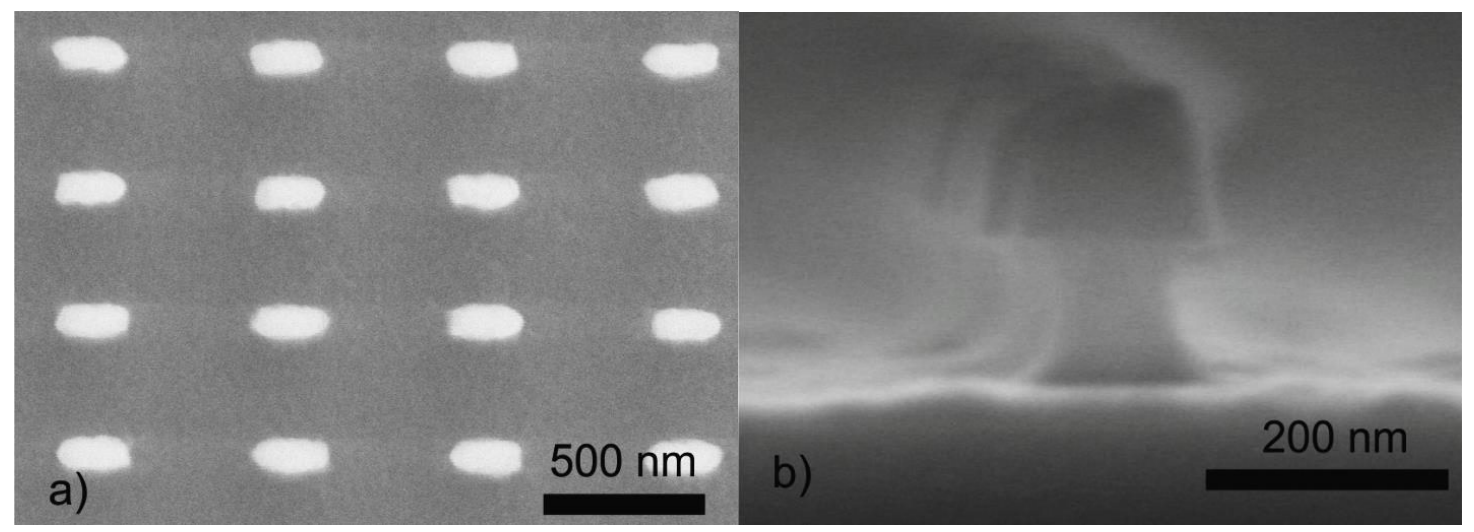

Figure 7: a) Dashes after CHF3 etching b) cross section SE image of a dash after BOE 100:1 for 35 sec showing the undercut profile. 
A $70 \mathrm{~nm}$ thick metallic layer $(\operatorname{Ti}(5 \mathrm{~nm}) / \mathrm{Au}(65 \mathrm{~nm}))$ was deposited by e-beam evaporation on each pattern. The samples were dipped in BOE 5:1 to perform the lift-off. Figure 8 displays the SE images taken after the lift-off. A metallic fishnet with period of 400nm and $600 \mathrm{~nm}$, with aperture of $120 \mathrm{~nm}$ in width and $290 \mathrm{~nm}$ in length were obtained for $50 / 20 \mathrm{~mJ} . \mathrm{cm}^{-2}$ combination and $105 \mathrm{~nm}$ in width and $210 \mathrm{~nm}$ in length for $50 / 25 \mathrm{~mJ} . \mathrm{cm}^{-2}$ combination.

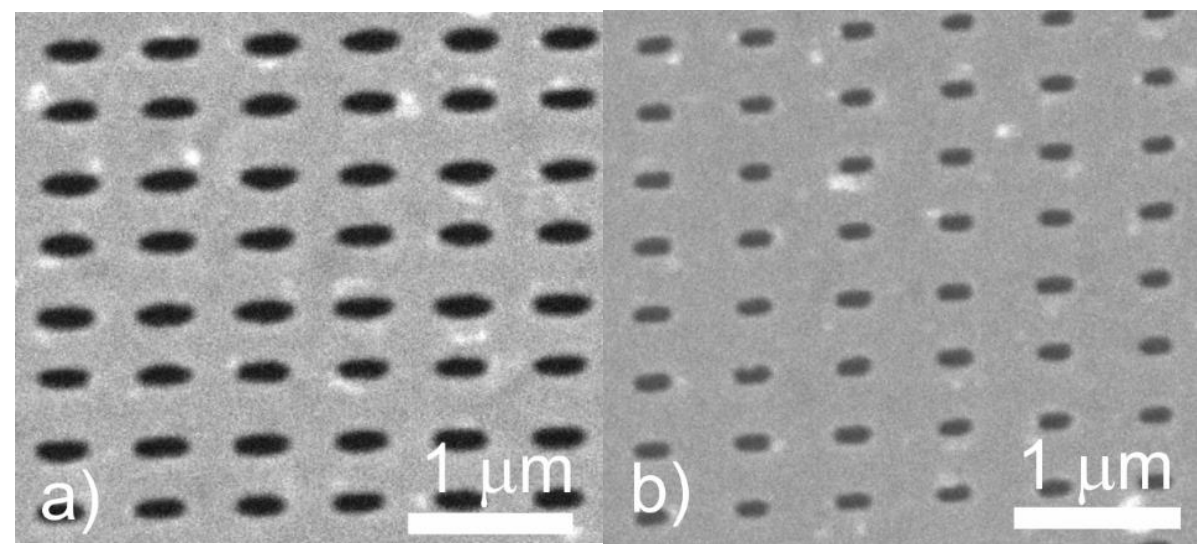

Figure 8: $\mathrm{Ti}(5 \mathrm{~nm}) / \mathrm{Au}(6 \mathrm{~nm})$ thick metallic 'fishnet' like structure obtained after lift-off for a dose of $50 \mathrm{~mJ} . \mathrm{cm}^{-2}$ for the $800 \mathrm{~nm}$ grating exposure and a dose of a) $20 \mathrm{~mJ} . \mathrm{cm}^{-2} \mathrm{~b}$ ) $25 \mathrm{~mJ}_{\mathrm{Jcm}} \mathrm{cm}^{-2}$ for the second $1.2 \mu \mathrm{m}$ grating exposure.

\subsection{Fabrication of elliptical holes in resist}

A similar process was performed using a $350 \mathrm{~nm}$ thick negative resist (AZ 15 NXT diluted with EBR about 7:12 by weight). Fig. 9 displays the secondary electron images of the resist pattern obtained for fixed exposure dose of $120 \mathrm{~mJ} . \mathrm{cm}^{-2}$ for the $800 \mathrm{~nm}$ period linear grating mask and a varied exposure dose of 35 to $50 \mathrm{~mJ} . \mathrm{cm}^{-2}$ for the $1.2 \mu \mathrm{m}$ period linear grating mask. Elliptical holes were obtained in the resist. The width and length of the elliptical holes decreased with the increase of the $1.2 \mu \mathrm{m}$ period linear grating exposure dose.

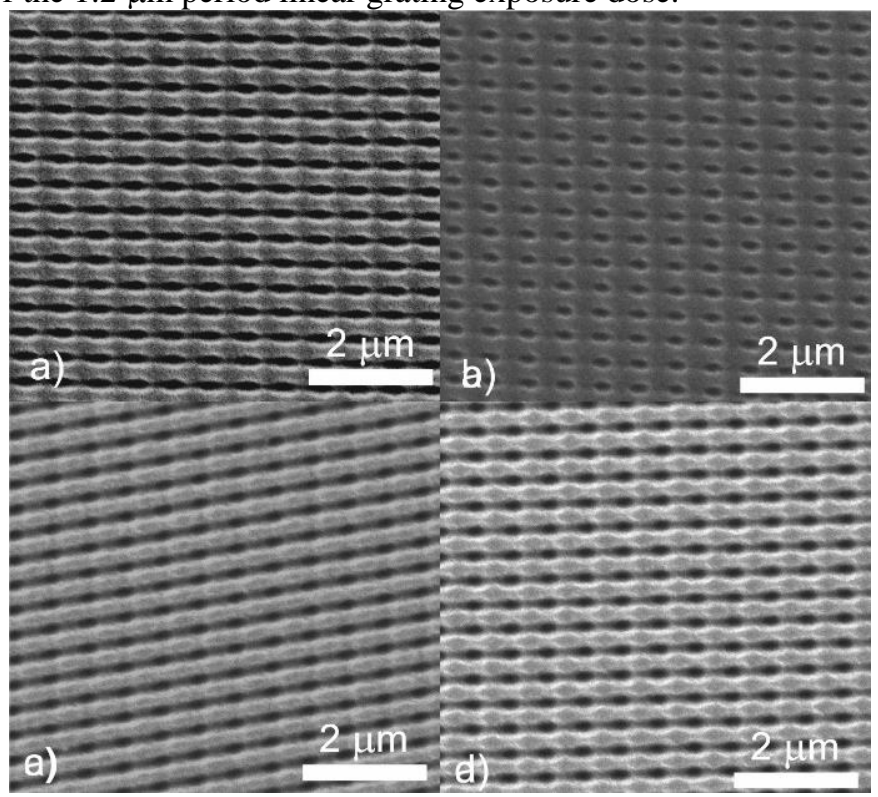

Figure 9: Elliptical holes obtained in a $350 \mathrm{~nm}$ thick negative resist after double exposure for a dose of $120 \mathrm{~mJ} . \mathrm{cm}^{-2}$ for the $800 \mathrm{~nm}$ linear grating pattern and a) $35 \mathrm{~mJ} . \mathrm{cm}^{-2}$ b) $40 \mathrm{~mJ} . \mathrm{cm}^{-2}$ c) $45 \mathrm{~mJ} . \mathrm{cm}^{-2}$ and d) $50 \mathrm{~mJ} . \mathrm{cm}^{-2}$ for the $1.2 \mu \mathrm{m}$ linear grating pitch 
The measured width (blue square) and length (red circle) of the holes are summarized and plotted in Fig. 10. The width and length of the holes are found to decrease with the increase of the second exposure dose (with $1.2 \mu \mathrm{m}$ period linear grating) in a similar fashion as shown previously for the resist dashes.

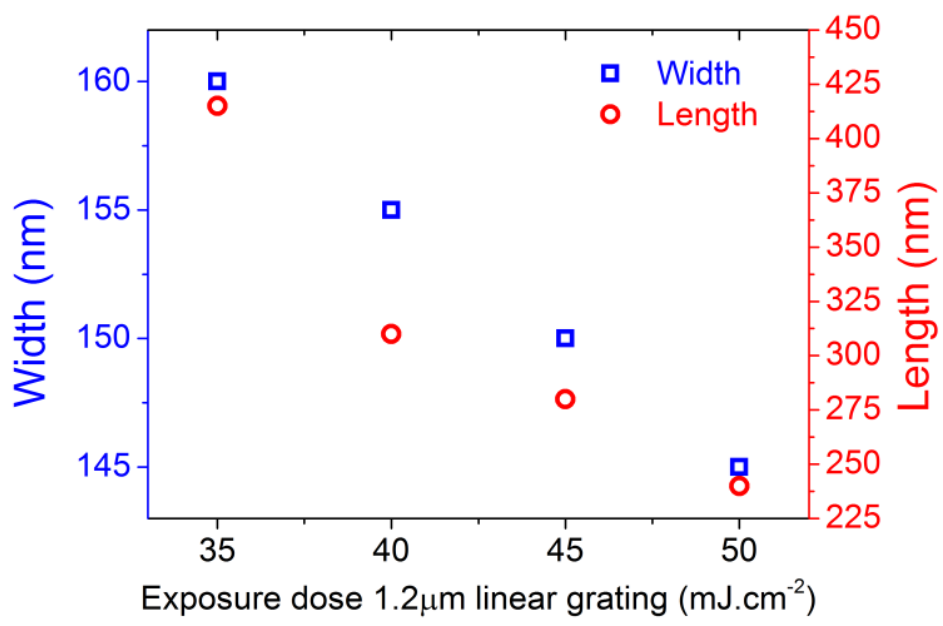

Figure 10: Length and width of the elliptical holes obtained for a fixed exposure dose of $120 \mathrm{~mJ}_{\mathrm{cm}} \mathrm{cm}^{-2}$ for the $800 \mathrm{~nm}$ period linear grating mask as a function of the dose for the $1.2 \mu \mathrm{m}$ period linear grating mask.

\section{CONCLUSION}

We have demonstrated that displacement Talbot lithography can be used to fabricate arrays of dashes and fishnet type structures with the dimensions that are required to fabricate negative-index metamaterials in the NIR range. The simplicity, high throughput, and low cost of such techniques combined with its high tunability range offers an interesting alternative to e-beam lithography or nanoimprint lithography techniques. In the future, we aim to further investigate the range of dimension that can be successfully fabricated and aim to demonstrate a lift-off process with the negative resist to obtain metallic or multilayer material dashes. We believe these techniques could pave the way to the fabrication of optical metamaterials and plasmonic metasurfaces in the near infrared and in the visible range in the near future.

\section{AUTHOR INFORMATION \& ACKNOWLEDGEMENTS}

* Corresponding Author: P.Shields@bath.ac.uk

The manuscript was written through contributions of all authors. / All authors have given approval to the final version of the manuscript.

Financial support is acknowledged from the EPSRC, UK via Grant No. EP/M015181/1, "Manufacturing nano-GaN". The data associated with this research are available at https://doi.org/10.15125/BATH-00363 or from the corresponding author.

\section{REFERENCES}

[1] Jiang, Z. H., Lin, L., Ma, D., Yun, S., Werner, D. H., Liu, Z.., Mayer, T. S., "Broadband and Wide Field-of-view Plasmonic Metasurface-enabled Waveplates.," Sci. Rep. 4, 7511 (2014).

[2] Zhang, Z., Luo, J., Song, M.., Yu, H., "Large-area, broadband and high-efficiency near-infrared linear polarization manipulating metasurface fabricated by orthogonal interference lithography," Appl. Phys. Lett. 107(24) (2015).

[3] Li, Z., Liu, W., Cheng, H., Chen, S.., Tian, J., "Realizing Broadband and Invertible Linear-to-circular Polarization Converter with Ultrathin Single-layer Metasurface,” Sci. Rep.(October 2015), 18106 (2015). 
[4] Xiao, S., Mühlenbernd, H., Li, G., Kenney, M., Liu, F., Zentgraf, T., Zhang, S.., Li, J., "Helicity-Preserving Omnidirectional Plasmonic Mirror,” Adv. Opt. Mater. 4(5), 654-658 (2016).

[5] Ross, M. B., Schatz, G. C., Sadeghi, S. M., Gutha, R. R., Wing, W. J.., Sharp, C., "Biological sensing and control of emission dynamics of quantum dot bioconjugates using arrays of long metallic nanorods," J. Phys. D Appl. Phys 50, 145401 (2017).

[6] Vecchi, G., Giannini, V.., Gómez Rivas, J., "Shaping the fluorescent emission by lattice resonances in plasmonic crystals of nanoantennas," Phys. Rev. Lett. 102(14), 2-5 (2009).

[7] Smythe, E. J., Cubukcu, E.., Capasso, F., "Optical properties of surface plasmon resonances of coupled metallic nanorods," Opt. Express 15(12), 7439-7447 (2007).

[8] Shorokhov, A. S., Okhlopkov, K. I., Reinhold, J., Helgert, C., Shcherbakov, M. R., Pertsch, T.., Fedyanin, A. A., "Ultrafast control of third-order optical nonlinearities in fishnet metamaterials," Sci. Rep. 6, 28440 (2016).

[9] Wang, L., Shorokhov, A. S., Melentiev, P. N., Kruk, S., Decker, M., Helgert, C., Setzpfandt, F., Fedyanin, A. A., Kivshar, Y. S., et al., "Multipolar Third-Harmonic Generation in Fishnet Metamaterials," ACS Photonics 3(8), 1494-1499 (2016).

[10] Van Nieuwstadt, J. A. H., Sandtke, M., Harmsen, R. H., Segerink, F. B., Prangsma, J. C., Enoch, S.., Kuipers, L., "Strong modification of the nonlinear optical response of metallic subwavelength hole arrays," Phys. Rev. Lett. 97(14), 1-4 (2006).

[11] Kim, E., Wang, F., Wu, W., Yu, Z.., Shen, Y. R., "Nonlinear optical spectroscopy of photonic metamaterials," Phys. Rev. B - Condens. Matter Mater. Phys. 78(11), 2-5 (2008).

[12] Shalaev, V. M., Cai, W., Chettiar, U. K., Yuan, H., Sarychev, A. K., Drachev, V. P.., Kildishev, A. V., "Negative index of refraction in optical metamaterials," Opt. Lett. 30(24), 3356-3358 (2005).

[13] Zhang, S., Fan, W., Panoiu, N. C., Malloy, K. J., Osgood, R. M.., Brueck, S. R. J., "Experimental demonstration of near-infrared negative-index metamaterials," Phys. Rev. Lett. 95(13), 1-4 (2005).

[14] Dolling, G., Enkrich, C., Wegener, M., Soukoulis, C. M.., Linden, S., "Simultaneous negative phase and group velocity of light in a metamaterial," Science (80-. ). 312, 892-894 (2006).

[15] Dolling, G., Enkrich, C., Wegener, M., Soukoulis, C. M.., Linden, S., "Low-loss negative-index metamaterial at telecommunication wavelengths," Opt. Lett. 31(12), 1800 (2006).

[16] Boltasseva, A.., Shalaev, V. M., "Fabrication of optical negative-index metamaterials: Recent advances and outlook," Metamaterials 2(1), 1-17 (2008).

[17] Dutta, N., Mirza, I. O., Shi, S.., Prather, D. W., "Fabrication of large area fishnet optical metamaterial structures operational at near-IR wavelengths," Materials (Basel). 3(12), 5283-5292 (2010).

[18] Dutta, N., Shi, S.., Prather, D. W., "Fabrication of large area 3D 'fishnet' optical metamaterial structures," Waves in Random and Complex Media 20(2), 289-297 (2010).

[19] Chanda, D., Shigeta, K., Gupta, S., Cain, T., Carlson, A., Mihi, A., Baca, A. J., Bogart, G. R., Braun, P., et al., "Large-area flexible 3D optical negative index metamaterial formed by nanotransfer printing," Nat. Nanotechnol. 6(7), 402-407 (2011). 
[20] Wu, W., Kim, E., Ponizovskaya, E., Liu, Y., Yu, Z., Fang, N., Shen, Y. R., Bratkovsky, A. M., Tong, W., et al., "Optical metamaterials at near and mid-IR range fabricated by nanoimprint lithography," Appl. Phys. A Mater. Sci. Process. 87(2), 143-150 (2007).

[21] Zhou, Y., Chen, X. Y., Fu, Y. H., Vienne, G., Kuznetsov, A. I.., Luk’Yanchuk, B., "Fabrication of large-area 3D optical fishnet metamaterial by laser interference lithography," Appl. Phys. Lett. 103(12), 1-5 (2013).

[22] Solak, H. H., Dais, C.., Clube, F., "Displacement Talbot lithography : a new method for high-resolution patterning of large areas," Opt. Express 19(11), 2530-2535 (2011).

[23] Wang, L., Clube, F., Dais, C., Solak, H. H.., Gobrecht, J., "Sub-wavelength printing in the deep ultra-violet region using Displacement Talbot Lithography," Microelectron. Eng. 161, 104-108 (2016).

[24] Solak, H. H., Dais, C., Clube, F.., Wang, L., "Phase shifting masks in Displacement Talbot Lithography for printing nano-grids and periodic motifs," Microelectron. Eng. 143, 74-80 (2015). 